\title{
Research and Simulation of the Electrical Vehicle Based Dynamical System
}

\author{
Ko-Chun Chen and Hsin-Yi Shih \\ Department of Applied Geoinformatics, Chia Nan University of Pharmacy \& Science, Tainan 717, Taiwan \\ Correspondence should be addressed to Hsin-Yi Shih; hsinyis@mail.chna.edu.tw
}

Received 1 June 2014; Accepted 4 September 2014

Academic Editor: Teen-Hang Meen

Copyright ( $) 2015$ K.-C. Chen and H.-Y. Shih. This is an open access article distributed under the Creative Commons Attribution License, which permits unrestricted use, distribution, and reproduction in any medium, provided the original work is properly cited.

This study developed a dynamic model of electric vehicle system by using the MATLAB/Simulink tool. The vehicle model comprises two system components: an electrical system and a suspension system. This study also designed various road conditions for simulating the motion of vehicle traveling along a road. The results show that the electrical and suspension system parameters can be adjusted immediately to enhance passenger comfort. The findings of this research have practical teaching applications. Students can modify the vehicle model parameters byes using the MATLAB graphical user interface, allowing them to observe the motion of vehicle under various road conditions.

\section{Introduction}

The automotive industry is well developed, and vehicle manufacturers are interested in producing cars that enhance passenger comfort. Therefore, various types of vehicle simulation model have been proposed, particularly those that enhance existing vehicle suspension systems. Recent studies have used MATLAB/Simulink to develop vehicle models for simulating how modifying the stiffness and damping value of a suspension system influences vehicle performance [1]. Another study used a frequency-adjusting method to control the vehicle suspension system [2]. A previous study on active suspension systems employed virtual instrumentation to test the performance of vehicle suspension systems, where PC-DAQ scheme was adopted to measure the absorptivity, vibration frequency, phase difference, and corresponding vibration waveform [3]. In addition, many recent studies have applied fuzzy controllers in their suspension system design. Previous studies have shown that vehicle suspension systems involving fuzzy controllers yield improved vehicle body stability during acceleration and displacement; compared with the systems featuring nonintegrated controllers, fuzzy controller-based systems yield a smaller overshoot curve. Several studies have shown that compared with nonintegrated controller-based systems, the peak acceleration and vehicle displacement can be reduced by at least $10 \%$ [46]. In other studies, neural network- and genetic algorithmbased fuzzy controllers have yielded superior vehicle suspension performance in comparison to systems that use conventional algorithm-based controllers [7]. In addition, fuzzy proportional-integral-derivative (PID) controllers have been applied widely in various types of vehicle control system. Fuzzy PID controllers markedly reduce the root mean square (RMS) pitch acceleration of the vehicle and RMS vertical acceleration of the passenger seat. Although fuzzy PID controllers produce simulation curves exhibiting relatively slower response speeds, their performance is relative to the performance of normal PID controllers that control the transition process, thereby reducing the time and overshoot considerably $[8,9]$. The aforementioned studies have used MATLAB to perform simulations. Several other studies have combined MATLAB with ADAMS to produce vehicle motion simulations that output motion data and produce animated vehicle simulations [10,11]. Moreover, most studies have discussed various methods for improving the performance of vehicle suspension systems to enhance passenger comfort. However, given the current emphasis on energy conservation and reducing the output of carbon emissions, the development of efficient electric vehicles is critical. Therefore, this study reviewed extant literature on hybrid electric vehicles 


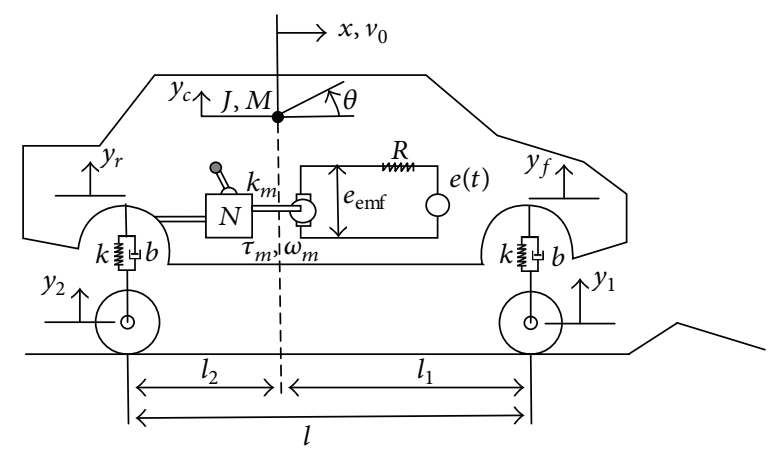

FIgURE 1: Model of the electric vehicle.

and vehicle simulations to design a simulation system for electric vehicles [10-12]. Vehicle motion is influenced by suspension system parameters, electrical system parameters, and road surface conditions. With the system proposed, in this study, the vehicle suspension system parameters can be adjusted through the MATLAB graphical user interface (GUI), and the corresponding effect on vehicle motion can be observed immediately.

\section{Dynamic Model of the Vehicle}

An electric vehicle moves from left to right at a constant longitudinal velocity $v$. With the suspension in a steady state, the front wheels encounter a constant slope input of $10 \%$ grade at time $t=0$. The rear wheels are subject to the same input as the front wheels with a time delay of $l / v_{0}$, where $l$ is the wheelbase of the vehicle. The vehicle body can be assumed to be a rigid body with a mass of $M$ and moment-ofinertia $J$. The response of the vehicle body can be expressed in terms of vertical position $y_{c}$ and counter clockwise rotation $\theta$ relative to the center of mass, which is located at a distance of $l_{1}$ from the front wheels and $l_{2}$ from the rear wheels. The front and rear suspensions can be modeled with identical springs of stiffness $k$ and identical dampers with damping coefficient $b$. First, we must obtain the vehicle model in the longitudinal, vertical, and pitch directions. Subsequently, various road conditions were designed to simulate the impact of a moving vehicle. Figure 1 depicts the overall vehicle model, and "Symbol Definition" section shows the electric vehicle model parameters. This study constructed a dynamic model of the vehicle shown in Figure 1 and detailed as follows.

2.1. Modeling of Dynamic Behavior in the Longitudinal Direction. In this section, we identify the relationship between the horizontal velocity and the applied voltage. In the longitudinal direction, the dynamic system includes the electrical and mechanical systems. Based on the model shown in Figure 1, the dynamic equations for the electrical system can be formulated as follows:

$$
\begin{aligned}
& e-i R-e_{\mathrm{emf}}, \\
& e_{\mathrm{emf}}=K_{b} w_{m} .
\end{aligned}
$$

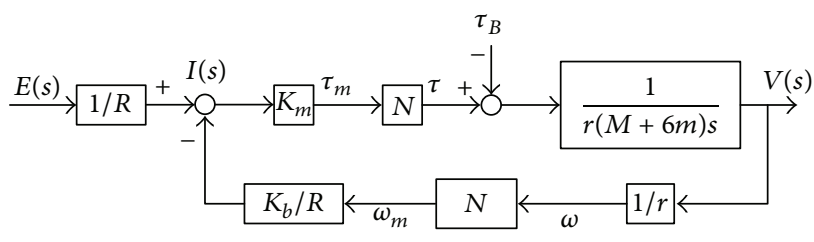

FIGURE 2: Control block diagram.

The motor equation can be expressed as

$$
\tau_{m}=K_{m} i
$$

The mechanical equation $\dot{w} \dot{x}$ is expressed as

$$
\begin{gathered}
\frac{n_{1}}{n_{2}}=\frac{r_{1}}{r_{2}}=\frac{w}{w_{m}}=\frac{1}{N} \\
{\left[J_{1}+J_{2}\left(\frac{n_{1}}{n_{2}}\right)^{2}\right] \dot{w}+\left[b_{1}+b_{2}\left(\frac{n_{1}}{n_{2}}\right)^{2}\right] w_{m}=\tau_{m}-\left(\frac{n_{1}}{n_{2}}\right) \tau .}
\end{gathered}
$$

According to the theory of energy conservation, when a vehicle is in motion, the kinetic and potential energy of the vehicle can be derived as follows:

$$
\begin{aligned}
T & =\frac{1}{2} M v^{2}+\frac{1}{2} \cdot(4 m) \cdot v^{2}+4 \cdot\left(\frac{1}{2} J w^{2}\right) \\
& =\frac{1}{2} M \dot{x}^{2}+\frac{1}{2}(4 m) \cdot \dot{x}^{2}+4 \cdot \frac{1}{2} \cdot \frac{1}{2} m r^{2} \cdot\left(\frac{\dot{x}}{r}\right)^{2} \\
& =\frac{1}{2}(M+6 m) \dot{x}^{2} .
\end{aligned}
$$

Subsequently, the energy method can be used to obtain the following:

$$
\frac{d}{d_{t}}(T+U)=0 \Longrightarrow(M+6 m) \ddot{x} .
$$

First, to identify the relationship between the longitudinal velocity and applied voltage, we must solve the transfer function $G(s)=V(s) / E(s)$. Second, (7) is obtained by taking the Laplace transform from (1), (2)-(4). Third, substitute (7) into (2) to obtain (8), as follows:

$$
\begin{gathered}
E(s)-I(s) R-K_{b} w_{m}=0 \Longrightarrow I(s)=\frac{E(s)-K_{b} w_{m}}{R}, \\
\tau_{m}=\frac{K_{m} E(s)-K_{b} K_{m} w_{m}}{R} .
\end{gathered}
$$

Thus, (8) is substituted into (4); subsequently, assume that no viscous friction occurs in each of the gear train components $b_{1}$ and $b_{2}$ (both are set with zero values) and that the motor inertia and load inertia referred to the motor shaft are negligible, implying that $J_{1}$ and $J_{2}$ are equal to zero. Finally, we obtain the transfer function shown in (9). According to (9), we construct the control block diagram shown in Figure 2. Consider

$$
\frac{V(s)}{E(s)}=\frac{r / N K_{b}}{\left[\left((M+6 m) R r^{2} / N^{2} K_{b} K_{m}\right) s+1\right]} .
$$




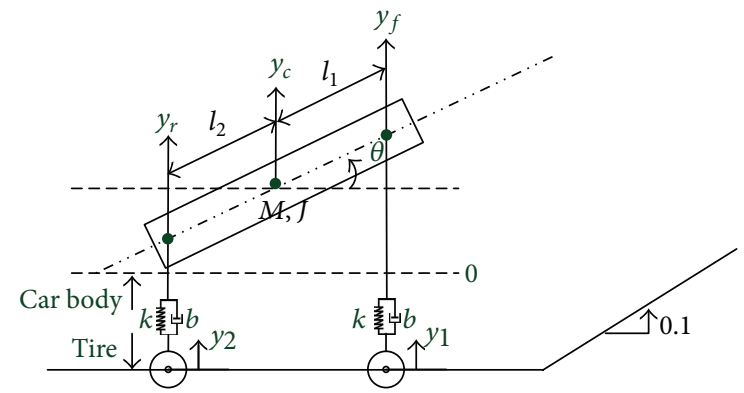

FIgURE 3: The vehicle vertical displacement and the pitch motion.

2.2. Modeling of Dynamic Behavior in the Vertical and Pitch Directions. In the second part, we must determine the vertical and pitch motion of the vehicle, as well as how the road conditions (i.e., system input) affect the vehicle motion. First, we simplify the vehicle model in Figure 1 as shown in Figure 3. According to Figure 3, Newton's second law is applied to determine the translational and rotational motion in $y_{c}$-direction and $\theta$-direction, respectively; thus, we obtain

$$
\begin{aligned}
M \ddot{y}_{c}= & 2 k\left(y_{1}-y_{f}\right)+2 k\left(y_{2}-y_{r}\right)+2 b\left(\dot{y}_{1}-\dot{y}_{f}\right) \\
& +2 b\left(\dot{y}_{2}-\dot{y}_{r}\right), \\
J \ddot{\theta}= & 2 k\left(y_{1}-y_{f}\right) l_{1}+2 k\left(y_{2}-y_{r}\right) l_{2}+2 b\left(\dot{y}_{1}-\dot{y}_{f}\right) l_{1} \\
+ & 2 b\left(\dot{y}_{2}-\dot{y}_{r}\right) l_{2} .
\end{aligned}
$$

Next, $y_{f}$ and $y_{r}$ are substituted with $y_{c}$ and $\theta$ as follows:

$$
\begin{aligned}
& y_{f}=y_{c}+l_{1} \theta, \\
& y_{r}=y_{c}-l_{2} \theta, \\
& \dot{y}_{f}=\dot{y}_{c}+l_{1} \theta, \\
& \dot{y}_{r}=\dot{y}_{c}-l_{2} \theta .
\end{aligned}
$$

Subsequently, we obtain

$$
\begin{aligned}
M \ddot{y}_{c}= & 2 k y_{1}-2 k\left(y_{c}+l_{1} \theta\right)+2 k y_{2}-2 k\left(y_{c}+l_{2} \theta\right)+2 b \dot{y}_{1} \\
& -2 b\left(\dot{y}_{c}+l_{1} \dot{\theta}\right)+2 b \dot{y}_{2}-2 b\left(\dot{y}_{c}+l_{2} \dot{\theta}\right), \\
j \ddot{\theta}= & 2 k l y_{1}-2 k l_{1}\left(y_{c}+l_{1} \theta\right)+2 k l_{2} y_{2}-2 k l_{2}\left(y_{c}+l_{2} \theta\right) \\
+ & 2 b l_{1} \dot{y}_{1}-2 b l_{1}\left(\dot{y}_{c}+l_{1} \dot{\theta}\right)+2 b l_{2} \dot{y}_{2} \\
& -2 b l_{2}\left(\dot{y}_{c}+l_{2} \dot{\theta}\right) .
\end{aligned}
$$

The function matrix for transferring the road profile to the car's vertical and pitch motions can be derived as

$$
\begin{aligned}
& M \ddot{y}_{c}+4 b \dot{y}_{c}+4 k y_{c}+2 b\left(l_{1}-l_{2}\right) \theta+2 k\left(l_{1}-l_{2}\right) \\
& =2\left(k y_{1}+b \dot{y}_{1}\right)+2\left(k y_{2}+b \dot{y}_{2}\right),
\end{aligned}
$$

$$
\begin{aligned}
j \ddot{\theta} & +2 b\left(l_{l}^{2}+l_{2}^{2}\right) \dot{\theta}+2 k\left(l_{l}^{2}+l_{2}^{2}\right) \theta+2 b\left(l_{1}-l_{2}\right) \dot{y}_{c} \\
& -2 k\left(l_{1}-l_{2}\right) y_{c}=2\left(k y_{1}+b \dot{y}_{1}\right) l_{1}-2\left(k y_{2}+b \dot{y}_{2}\right) l_{2} .
\end{aligned}
$$

Subsequently, take the Laplace transform

$$
\begin{aligned}
& \left(M s^{2}+4 b s+4 k\right) Y_{c}(s)+2\left[b\left(l_{1}-l_{2}\right) s+k\left(l_{1}-l_{2}\right)\right] \theta(s) \\
& =2(b s+k) Y_{1}(s)+2(b s+k) Y_{2}(s), \\
& 2\left[b\left(l_{1}-l_{2}\right) s+k\left(l_{1}-l_{2}\right)\right] Y_{c}(s) \\
& \quad+\left[j s^{2}+2 b\left(l_{1}^{2}+l_{2}^{2}\right) s+2 k\left(l_{1}^{2}+l_{2}^{2}\right)\right] \theta(s) \\
& =2(b s+k) l_{1} Y_{1}(s)-2(b s+k) l_{2} Y_{2}(s) .
\end{aligned}
$$

Finally, we obtain the matrix of the transfer function

$$
\left[\begin{array}{c}
Y_{c}(s) \\
\theta(s)
\end{array}\right]=\left[\begin{array}{ll}
G_{11} & G_{12} \\
G_{21} & G_{22}
\end{array}\right]\left[\begin{array}{c}
Y_{1}(s) \\
Y_{2}(s)
\end{array}\right]
$$

where $G_{11}, G_{12}, G_{21}$, and $G_{22}$ are expressed as follows:

$$
\begin{aligned}
& G_{11} \\
& =\left(\left[J s^{2}+2 b\left(l_{1}^{2}+l_{2}^{2}\right) s+2 k\left(l_{1}^{2}+l_{2}^{2}\right)\right] 2(b s+k)\right. \\
& \left.-2\left[b\left(l_{1}-l_{2}\right) s+k\left(l_{1}-l_{2}\right)\right] 2(b s+k) l_{1}\right) \\
& \times\left(\left(M s^{2}+4 b s+4 k\right)\left[J s^{2}+2 b\left(l_{1}^{2}+l_{2}^{2}\right) s+2 k\left(l_{1}^{2}+l_{2}^{2}\right)\right]\right. \\
& \left.-4\left[b\left(l_{1}-l_{2}\right) s+k\left(l_{1}-l_{2}\right)\right]^{2}\right)^{-1}, \\
& G_{12} \\
& =\left(\left[J s^{2}+2 b\left(l_{1}^{2}+l_{2}^{2}\right) s+2 k\left(l_{1}^{2}+l_{2}^{2}\right)\right] 2(b s+k)\right. \\
& \left.-2\left[b\left(l_{1}-l_{2}\right) s+k\left(l_{1}-l_{2}\right)\right] 2(b s+k) l_{2}\right) \\
& \times\left(\left(M s^{2}+4 b s+4 k\right)\left[J s^{2}+2 b\left(l_{1}^{2}+l_{2}^{2}\right) s+2 k\left(l_{1}^{2}+l_{2}^{2}\right)\right]\right. \\
& \left.-4\left[b\left(l_{1}-l_{2}\right) s+k\left(l_{1}-l_{2}\right)\right]^{2}\right)^{-1}, \\
& G_{21} \\
& =\left(-2\left[b\left(l_{1}-l_{2}\right) s+k\left(l_{1}-l_{2}\right)\right] 2(b s+k)\right. \\
& \left.+\left(M s^{2}+4 b s+4 k\right) 2(b s+k) l_{1}\right) \\
& \times\left(\left(M s^{2}+4 b s+4 k\right)\left[J s^{2}+2 b\left(l_{1}^{2}+l_{2}^{2}\right) s+2 k\left(l_{1}^{2}+l_{2}^{2}\right)\right]\right. \\
& \left.-4\left[b\left(l_{1}-l_{2}\right) s+k\left(l_{1}-l_{2}\right)\right]^{2}\right)^{-1}, \\
& G_{22} \\
& \begin{aligned}
=( & -2\left[b\left(l_{1}-l_{2}\right) s+k\left(l_{1}-l_{2}\right)\right] 2(b s+k) \\
& \left.+\left(M s^{2}+4 b s+4 k\right) 2(b s+k) l_{2}\right) \\
\times & \left(\left(M s^{2}+4 b s+4 k\right)\left[J s^{2}+2 b\left(l_{1}^{2}+l_{2}^{2}\right) s+2 k\left(l_{1}^{2}+l_{2}^{2}\right)\right]\right. \\
& \left.-4\left[b\left(l_{1}-l_{2}\right) s+k\left(l_{1}-l_{2}\right)\right]^{2}\right)^{-1} .
\end{aligned}
\end{aligned}
$$




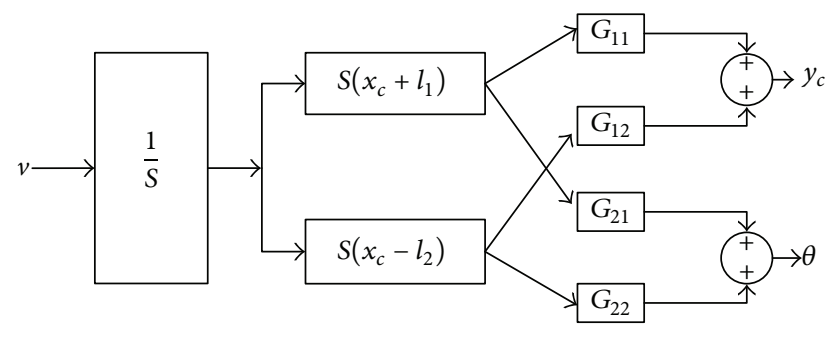

FIGURE 4: The vertical displacement and rotational motion and the longitudinal velocity.

From (17), we know that the inputs are $Y_{1}(s)$ and $Y_{2}(s)$, which are produced by the road profile $S\left(x_{c}\right)$. Therefore, we can obtain the block diagram control of the vertical displacement $y_{c}$ and longitudinal velocity $v$. The rotational motion $\theta$ and longitudinal velocity $v$ correspond with the road input, as shown in Figure 4.

2.3. State-Space Equation of the Vehicle. The state-space equation of the vehicle is derived to obtain the acceleration, angular velocity, and angular acceleration of the vehicle. From (11) and (13), assuming that $z_{1}=y_{c}, \dot{z}_{1}=\dot{y}_{c}=z_{2}, \dot{z}_{2}=\ddot{y}_{c}$, $z_{3}=\theta, \dot{z}_{3}=\dot{\theta}=z_{4}$, and $\dot{z}_{4}=\ddot{\theta}$, then the state-space equation can be obtained as follows:

$$
\begin{aligned}
& {\left[\begin{array}{l}
\dot{Z}_{1} \\
\dot{Z}_{2} \\
\dot{Z}_{3} \\
\dot{Z}_{4}
\end{array}\right]} \\
& =\left[\begin{array}{cccc}
0 & 1 & 0 & 0 \\
\frac{-4 k}{m} & \frac{-4 b}{m} & \frac{-2 k\left(l_{1}-l_{2}\right)}{m} & \frac{-2 b\left(l_{1}-l_{2}\right)}{m} \\
0 & 0 & 0 & 1 \\
\frac{-2 k\left(l_{1}-l_{2}\right)}{J} & \frac{-2 b\left(l_{1}-l_{2}\right)}{J} & \frac{-2 k\left(l_{1}^{2}-l_{2}^{2}\right)}{J} & \frac{-2 b\left(l_{1}^{2}-l_{2}^{2}\right)}{J}
\end{array}\right] \\
& \times\left[\begin{array}{l}
Z_{1} \\
Z_{2} \\
Z_{3} \\
Z_{4}
\end{array}\right] \\
& +\left[\begin{array}{cccc}
0 & 0 & 0 & 0 \\
\frac{2 k}{m} & \frac{2 b}{m} & \frac{2 k}{m} & \frac{2 b}{m} \\
0 & 0 & 0 & 0 \\
\frac{2 k l_{1}}{J} & \frac{2 b l_{1}}{J} & \frac{-2 k l_{2}}{J} & \frac{-2 b l_{2}}{J}
\end{array}\right]\left[\begin{array}{c}
y_{1} \\
\dot{y}_{1} \\
y_{2} \\
\dot{y}_{2}
\end{array}\right]
\end{aligned}
$$

where the output is

$$
\left[\begin{array}{c}
y_{c} \\
\dot{y}_{c} \\
\theta \\
\dot{\theta}
\end{array}\right]=\left[\begin{array}{llll}
1 & 0 & 0 & 0 \\
0 & 1 & 0 & 0 \\
0 & 0 & 1 & 0 \\
0 & 0 & 0 & 1
\end{array}\right]\left[\begin{array}{l}
z_{1} \\
z_{2} \\
z_{3} \\
z_{4}
\end{array}\right],
$$

where $z_{1}$ and $z_{3}$ denote the vertical position and pitch angle of vehicle, respectively; state variables $z_{2}$ and $z_{4}$ are the time derivatives of $z_{1}$ and $z_{3}$, respectively; $y_{c}, \dot{y}_{c}, \theta$, and $\dot{\theta}$ are the output variables corresponding to $z_{1}, z_{2}, z_{3}$, and $z_{4}$, respectively.

\section{Simulation Result and Analysis}

The proposed dynamic model of an electric vehicle was developed using MATLAB/Simulink. The simulation conditions are detailed as follows: $m=1310 \mathrm{~kg}, l_{1}=1 \mathrm{~m}, l_{2}=1 \mathrm{~m}, k=$ $12929 \mathrm{~N} / \mathrm{m}, b=2880 \mathrm{~N}-\mathrm{s} / \mathrm{m}, K_{b}=14 \mathrm{~V}-\mathrm{s} / \mathrm{rad}, K_{m}=10 \mathrm{~N}-$ $\mathrm{m} / \mathrm{A}, N=20$, and $R=5 \mathrm{k} \Omega$. This section comprises three parts. The first part reports the influence of the applied voltage on the longitudinal velocity of the vehicle. The second part analyzes the influence of input road conditions on the vertical displacement and pitch angle of the vehicle. Finally, the third part constructs a dynamic model of the state-space and then simulates and analyzes the vehicle motion in the vertical and pitch directions.

3.1. Influence of the Applied Voltage on the Longitudinal Velocity of the Vehicle. Assume that the vehicle travels on a flat road surface free of obstacles. According to (8), we set the applied voltage to $12,24,36$, and $48 \mathrm{~V}$ and then calculate the results of the longitudinal velocity, which increases in conjunction with the voltage, and the final velocity is 6.4, 12.9, 19.3 , and $25.7 \mathrm{~m} / \mathrm{s}$, respectively. Simultaneously, the vehicle's longitudinal displacement is also increased. Figure 5 shows the results.

\subsection{Influence of the Input Road Conditions on the Longitudinal} Displacement and Pitch Angle of the Vehicle. According to Figure 4, assume that the vehicle travels on the road at a velocity of $12.9,19.4$, and $27.8 \mathrm{~m} / \mathrm{s}$, and an identical displacement is applied from the center of mass to the front and rear wheels; in other words, $l_{1}=l_{2}=1 \mathrm{~m}$. After the vehicle travels a distance of $1 \mathrm{~km}$, the road conditions become bumpy, and the slopes of the uphill and downhill path are 0.1 and -0.2 , respectively (Figure 6). Figure 7 depicts the vertical displacement and pitch angle of the vehicle as it travels along the bumpy road surface. As shown in Figure 7, at the point where the vehicle travels from a flat road surface onto a sloped road surface (or vice versa), its variation in displacement and angular displacement increase. Moreover, the increase is more pronounced when travelling downhill because of the impact of gravity. Because the velocity of vehicle is higher, the rear wheels become raised when the vehicle meets the flat road; hence, the vehicle exhibits negative displacement and angular displacement.

3.3. Vehicle Motion and State-Space. According to the aforementioned results, the vehicle acceleration cannot be obtained directly; therefore, we adjusted the equation of motion relative to the state-space form. For the simulation, the simulation time was set at $3 \mathrm{~s}$; the velocity of vehicle $v$ was set at 11.1, 19.4, and $27.8 \mathrm{~m} / \mathrm{s}$; and the road slope was set at 0.1 . Finally, Figure 8 shows the acceleration, velocity, and displacement of the vehicle in vertical and pitch directions. As shown in Figure 8, when the vehicle velocity 

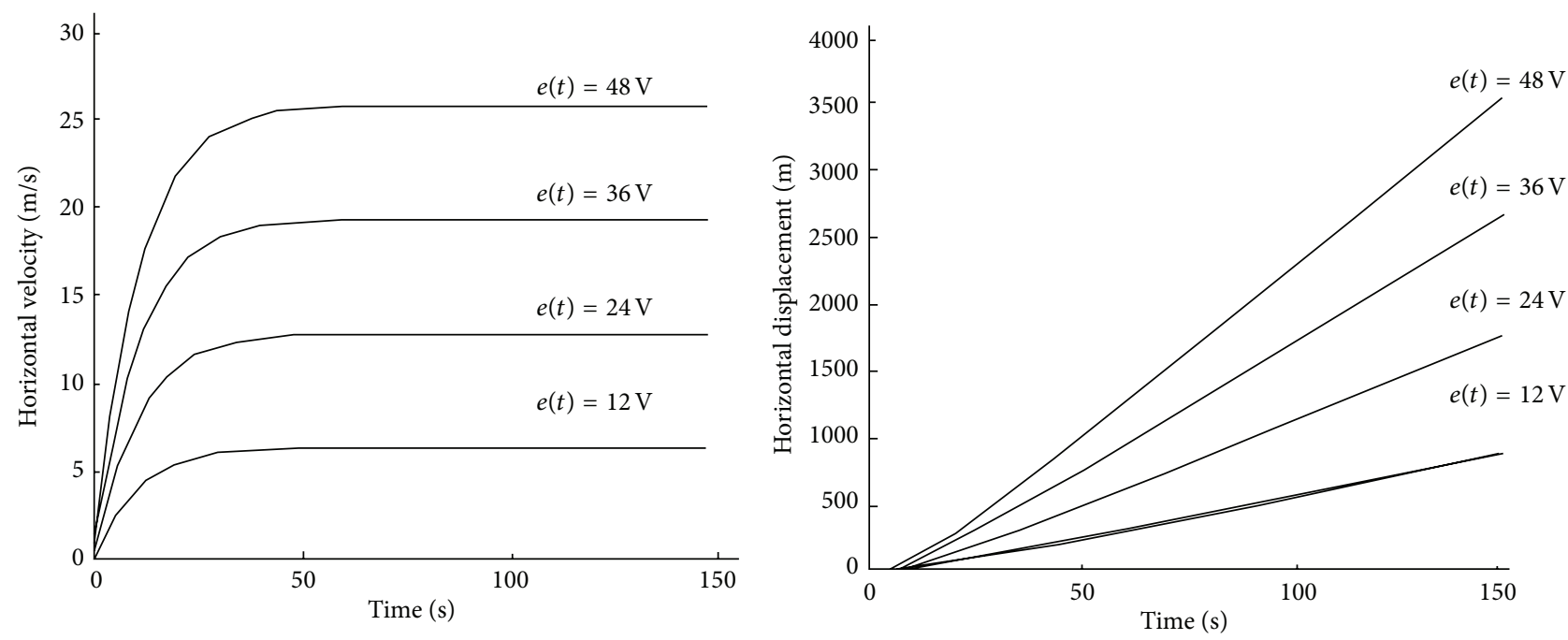

FIGURE 5: The longitudinal velocity and the longitudinal displacement of the vehicle.

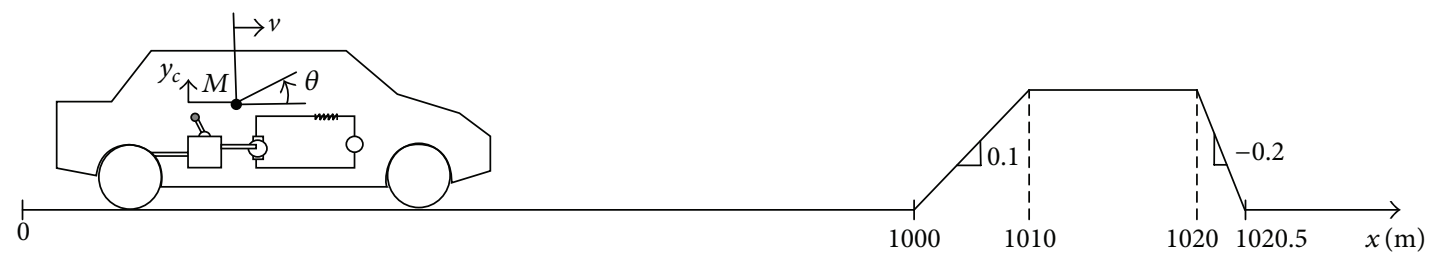

FIGURE 6: The vehicle is through a bumpy, and then the center of vehicle produce the vertical displacement and pitch rotation as shown in Figure 7.
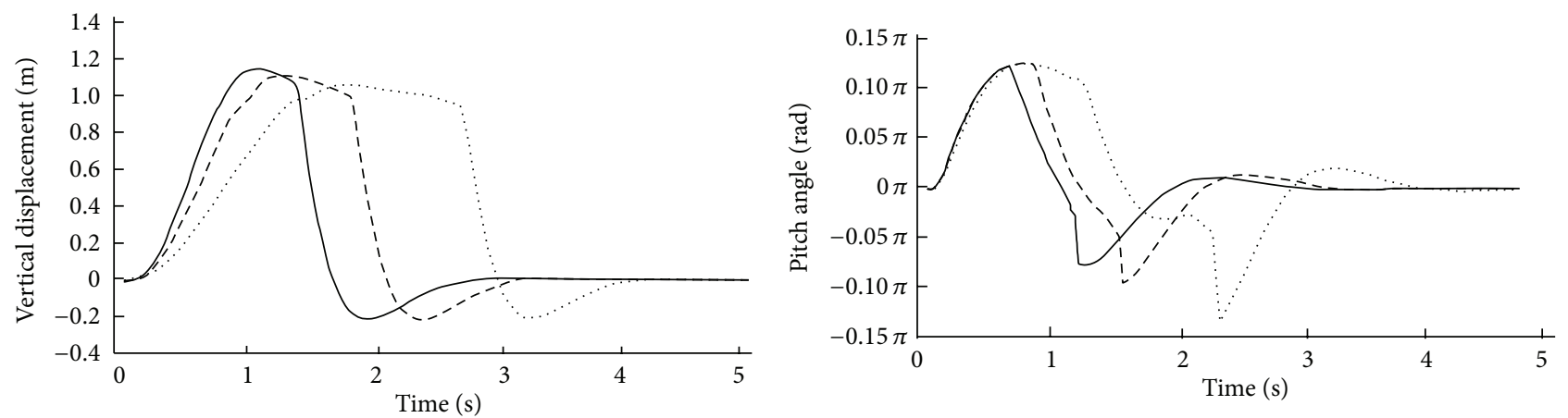

FIGURE 7: The vertical displacement and the pitch angle when it is through a bumpy road.

is higher, it produces more acceleration; in particular, at the moment when the vehicle begins travelling uphill, its body tilts forward slightly, thereby causing the rear wheels to be raised; consequently, the vehicle exhibits negative acceleration and angular acceleration. After $1.5 \mathrm{~s}$, the vehicle enters a steady state and its angular displacement converges to $0.1 \pi$, which is the slope of uphill path. Second, the vehicle acceleration, angular velocity, and displacement are increased; consequently, the vehicle requires more time to return to a steady state.

\section{Graphic User Interface}

Most previous studies have used only MATLAB/Simulink to construct vehicle models. However, this study employed MATLAB/Simulink to construct the vehicle model and a GUI to allow interaction with the Simulink model. As shown in Figure 9, the vehicle parameters can be modified, and the results can be observed immediately through the GUI. Thus, the function of the road conditions can be developed by interacting with the Simulink Stateflow, which is a flow-controlled 


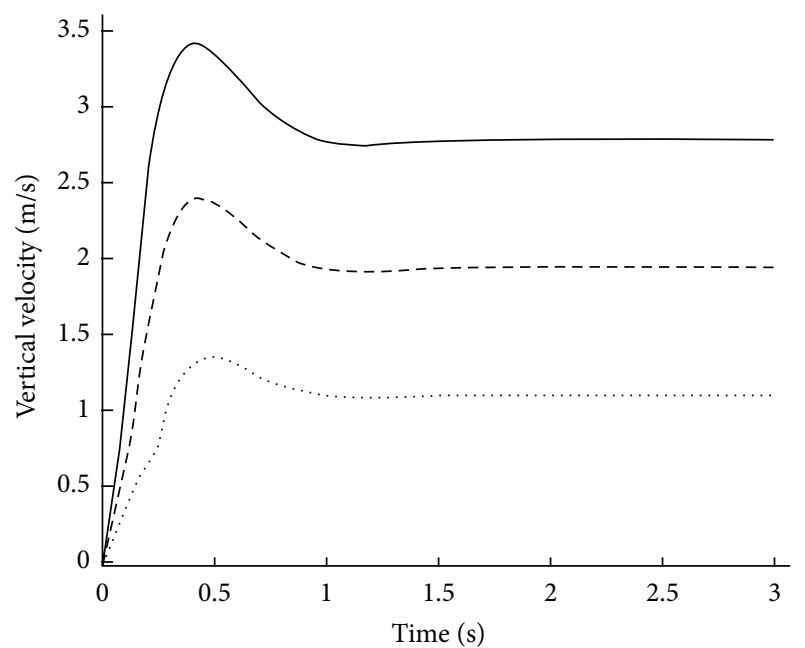

(a)

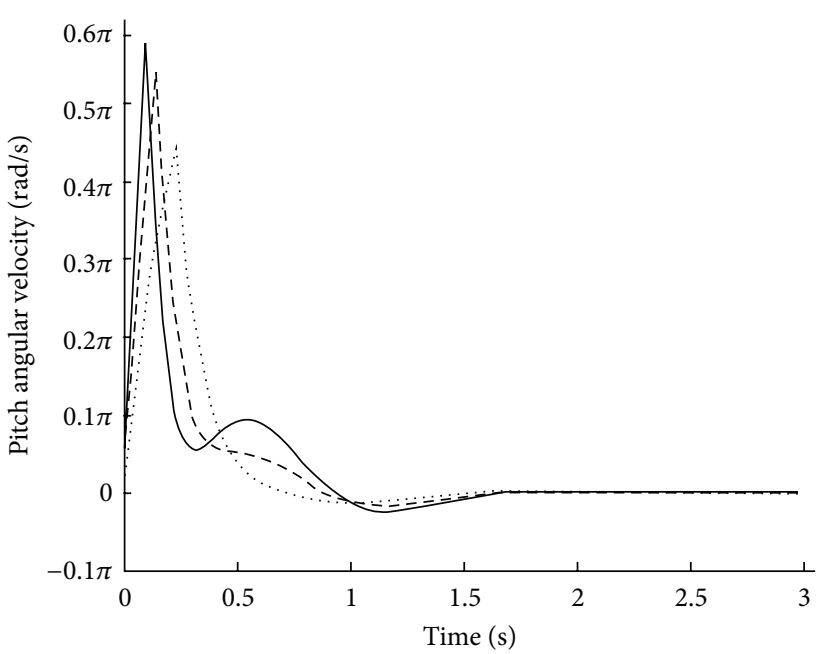

(b)

FIGURE 8: The motion of vehicle in vertical and pitch direction.
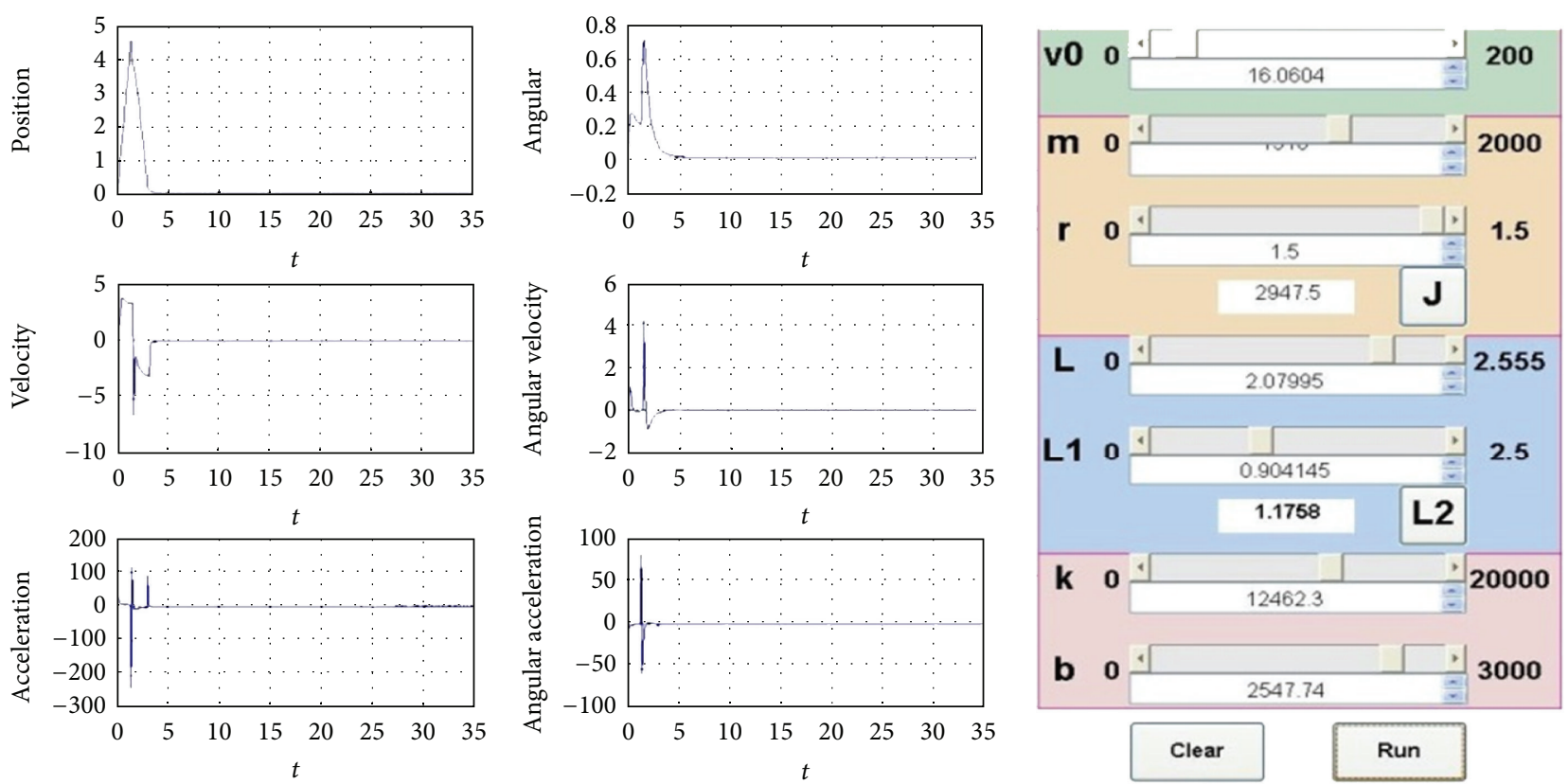

FIGURE 9: One can adjust the parameters of vehicle by GUI and observe the motion of vehicle, such as displacement, velocity, and acceleration of vertical and pitch direction.

block. Operators can use the Stateflow block to control the system flow and to link multiple systems simultaneously. Furthermore, this research design can be used to develop sloped or bumpy road conditions, as well as trapezoidal roads, or other types of road functions by using the Stateflow block, as shown in Figure 10; subsequently, the road functions can be linked with vehicle control system. In this case, we can observe the vehicle motion while the vehicle travels along various road types. Furthermore, this research can be applied to instruct students by adjusting the vehicle parameters, such as the mass, spring constant, damping coefficient, and wheelbase length. Furthermore, the variation in vehicle motion (e.g., displacement, velocity, and acceleration along the vertical, longitudinal, and pitch directions) can be observed.

\section{Conclusions}

According to the results of this study, the longitudinal velocity of the vehicle depends on the applied voltage input. When the vehicle travels along a road that is free of obstacles, the applied voltage and longitudinal velocity are higher. Under contrasting conditions, these values are lower. In general, the maximal applied voltage input is $48 \mathrm{~V}$; accordingly, we can obtain the final longitudinal velocity of $25.7 \mathrm{~m} / \mathrm{s}$. In addition, 


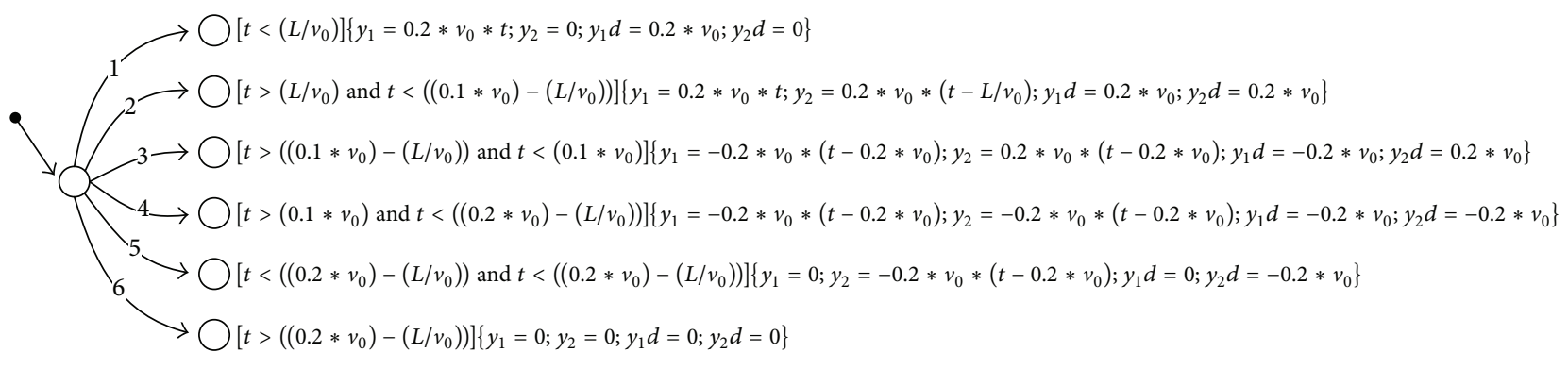

Figure 10: The road input is designed by using Stateflow of MATLAB.

the vertical displacement and pitch angle are determined by the input road conditions. If the input road is bumpy, the vertical displacement and pitch angle are higher. Similarly, under contrasting conditions, these values are lower. Finally, the peak acceleration or angular acceleration occurs at the moment when the vehicle travels from a flat road onto an uphill path (or when it travels from a downhill path onto a flat road). This study used MATLAB/Simulink to design an electric vehicle system that is suitable for instructional applications. The vehicle parameters can be adjusted conveniently, and the variation in vehicle motion can be observed immediately; thus, engineers or students can modify the vehicle parameters in real time.

\section{Symbol Definition}

$b$ : Damping coefficient

$e(t)$ : Applied voltage to the drive motor

$e_{\text {emf }}$ : Back emf

J: Moment-of-inertia of the vehicle body

$k$ : $\quad$ Spring constant

$K_{m}$ : Motor torque constant

$K_{b}$ : Back emf constant

$l$ : Length of the wheel base

$l_{1}$ : Distance from front wheel to center of vehicle

$l_{2}$ : Distance from rear wheel to center of vehicle

$M$ : Center of mass of the vehicle

$m$ : Mass of a wheel

$N$ : Gear ratio of gear box

$R: \quad$ Resistance of the motor coil

$r$ : Radius of the wheels

$v$ : Longitudinal velocity of the vehicle

$x$ : Longitudinal displacement of the vehicle

$y_{c}$ : Vertical displacement of the center of vehicle

$y_{f}$ : Vertical displacement of the front wheel

$y_{r}$ : Vertical displacement of the rear wheel

$y_{1}$ : Input road to the front wheels

$y_{2}$ : Input road to the rear wheels

$\theta$ : Counter clockwise rotation of the vehicle.

\section{Conflict of Interests}

The authors declare that there is no conflict of interests regarding the publication of this paper.

\section{References}

[1] B. Lu, S. Zhu, and Y. Zhang, Proceedings of the IEEE International Conference on Automation and Logistics, Jinan, China, 2007.

[2] X. Guan, S. Jian, Z. Libin, G. Haibin, and X. Xiangguo, "Research on automobile suspension performance evaluation system with adjusting-frequency method," in Proceedings of the International Conference on Research Challenges in Computer Science (ICRCCS '09), pp. 202-205, Shanghai, China, December 2009.

[3] Y. Liu, X. Cao, L. Cui, and W. Xiong, "Vehicle suspensions performance testing system based on virtual instrument," in Proceedings of the IEEE International Conference on Mechatronics and Automation (ICMA '09), pp. 605-610, Changchun, China, August 2009.

[4] S. Arabi and M. Behroozi, "Design of an integrated active front steering and active rear differential controller using fuzzy logic control," in Proceedings of the World Congress on Engineering (WCE '10), vol. 2, pp. 1409-1414, London, UK, June-July 2010.

[5] Z. Yinhuan, in Proceedings of the International Conference on Mechanic Automation and Control Engineering, Wuhan China, June 2010.

[6] H. Xiao, W. Chen, H. Zhou, and J. W. Zu, "Integrated vehicle dynamics control through coordinating electronic stability program and active suspension system," in Proceedings of the IEEE International Conference on Mechatronics and Automation (ICMA '09), pp. 1150-1155, Changchun, China, August 2009.

[7] J. Cao, H. Liu, P. Li, and D. J. Brown, "State of the art in vehicle active suspension adaptive control systems based on intelligent methodologies," IEEE Transactions on Intelligent Transportation Systems, vol. 9, pp. 392-405, 2008.

[8] S. Jianmin, W. Yuejin, and L. Huanying, "Comparative study on vibration control of engineering vehicle suspension system," in Proceedings of the International Conference on Intelligent Computation Technology and Automation, pp. 989-992, Beijing, China, May 2010.

[9] T. Lingfeng and L. Shengwe, "PID-fuzzy control research on hydraulic semi-active suspension system of car," in Proceedings of the International Conference on Educational and Network Technology, pp. 163-166, Qinhuangdao, China, June 2010.

[10] D. Kim, S. Hwang, and H. Kim, "Vehicle stability enhancement of four-wheel-drive hybrid electric vehicle using rear motor control," IEEE Transactions on Vehicular Technology, vol. 57, no. 2, pp. 727-735, 2008.

[11] Y. Zhang, M. M. Xia, J. Y. Qin, and H. Zhang, "Research on co-simulation using ADAMS and MATLAB for automobile active suspension system," in Proceedings of the International 
Conference on Computer Application and System Modeling (ICCASM '10), vol. 14, pp. 366-370, Taiyuan, China, October 2010.

[12] L. Nehaoua, A. Amouri, and H. Arioui, in Proceedings of the 13th Mediterranean Conference on Control and Automation, Limassol, Cyprus, June 2005. 


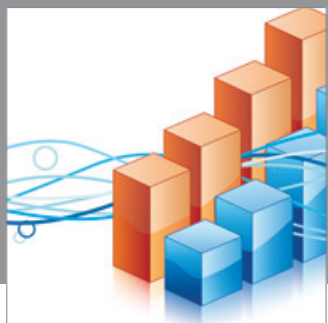

Advances in

Operations Research

mansans

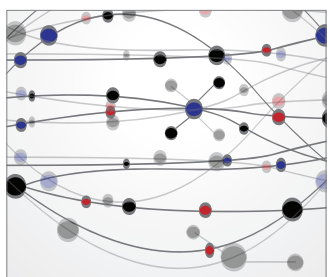

The Scientific World Journal
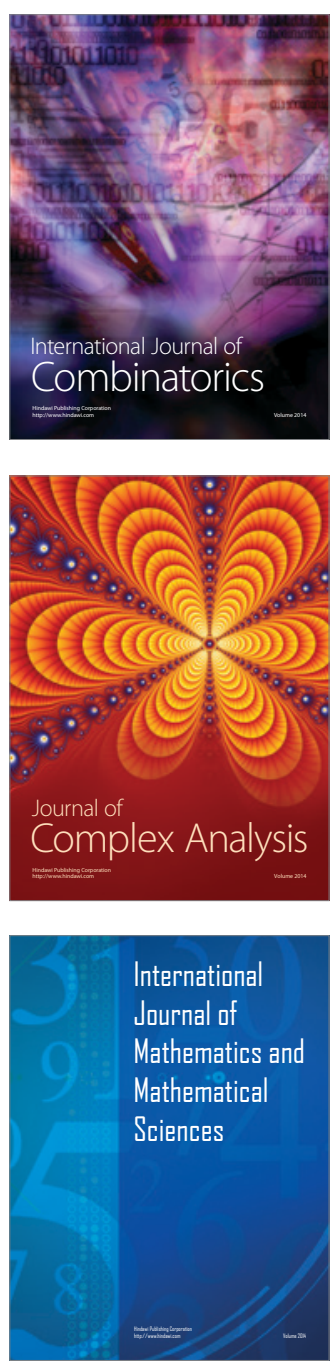
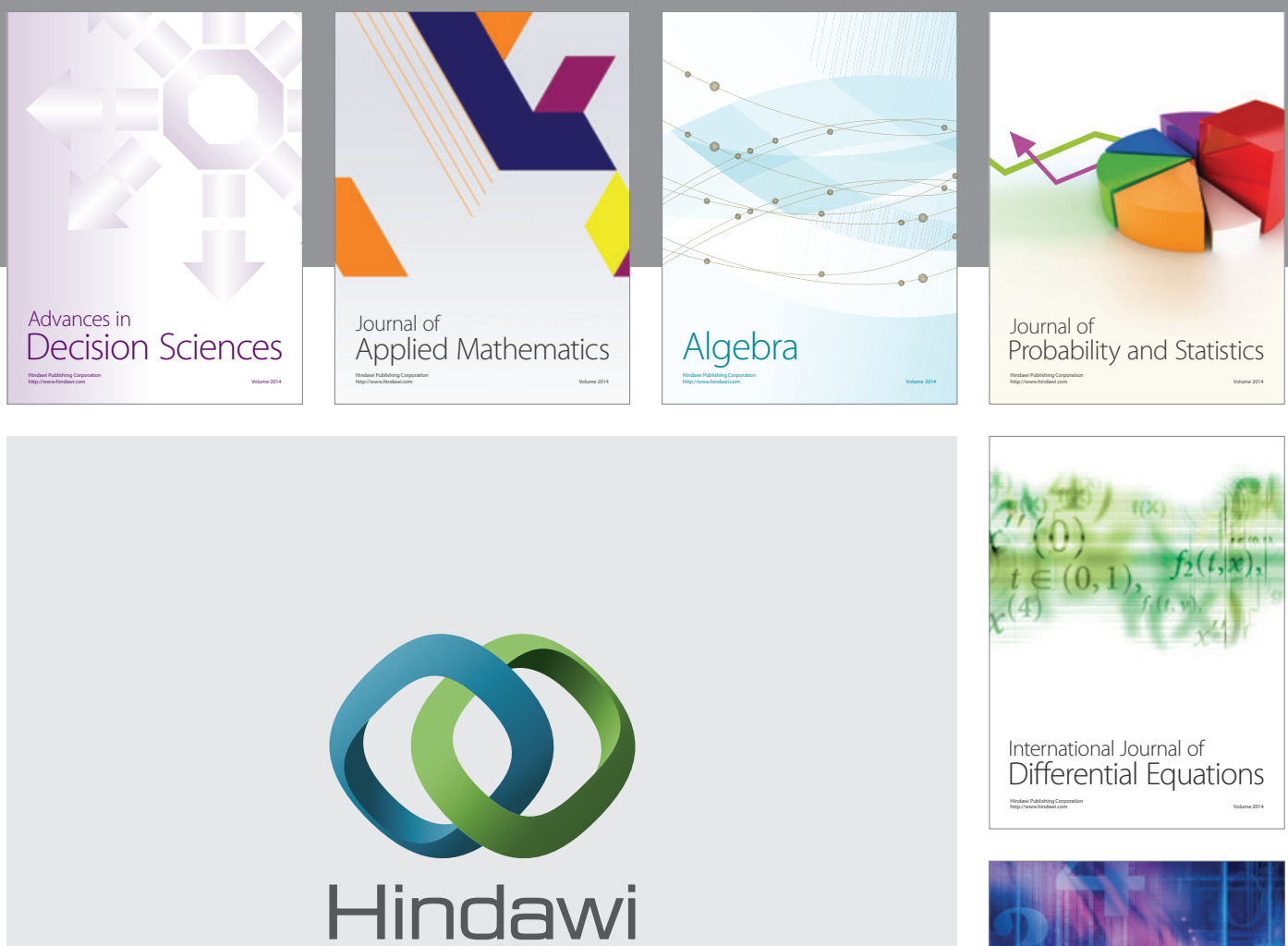

Submit your manuscripts at http://www.hindawi.com
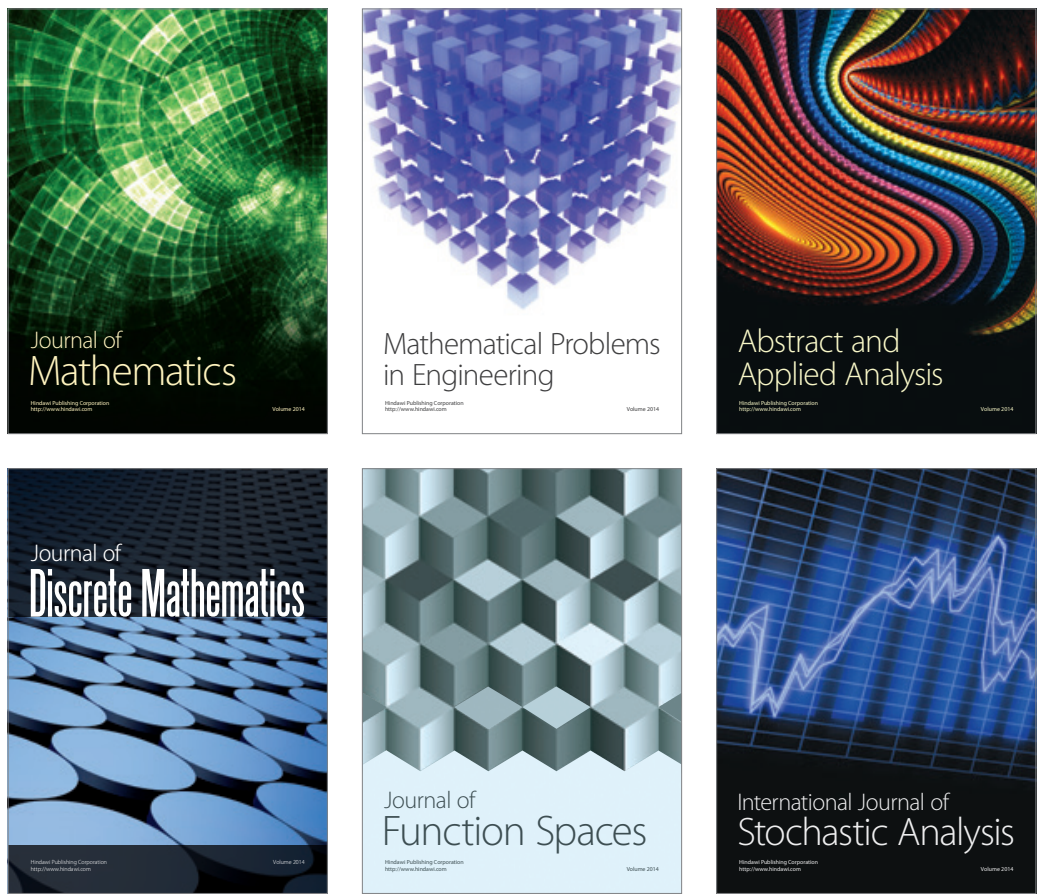

Journal of

Function Spaces

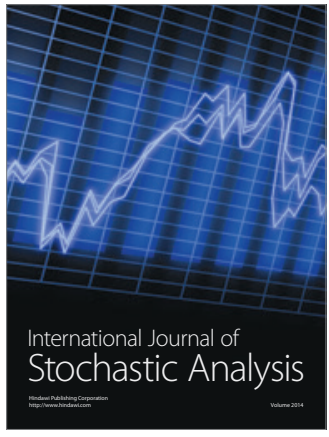

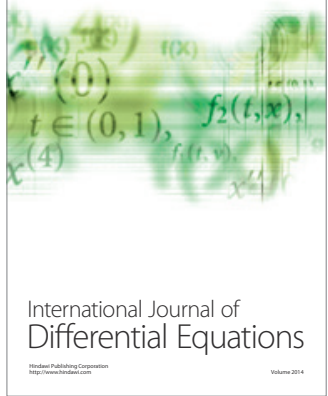
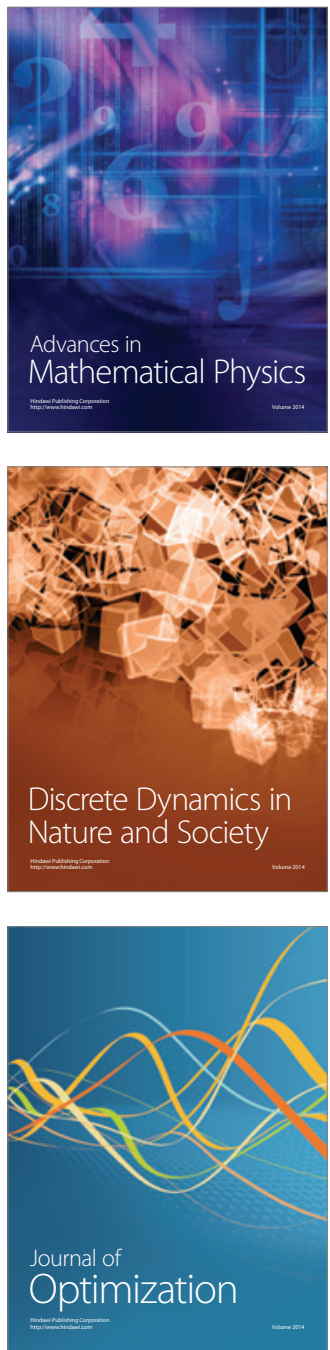